\title{
Seroprevalence survey on measles, mumps, rubella and varicella antibodies in healthcare workers in Japan: sex, age, occupational-related differences and vaccine efficacy
}

\author{
S. KUMAKURA ${ }^{1,2 *}$, H. SHIBATA ${ }^{2,3}$, K. ONODA $^{4}$, N. NISHIMURA ${ }^{2,5}$, \\ C. MATSUDA ${ }^{3}$ AND M. HIROSE ${ }^{2,6}$ \\ ${ }^{1}$ Department of Medical Education and Research; ${ }^{2}$ Infection Control Committee; ${ }^{3}$ Department of Laboratory \\ Medicine; ${ }^{4}$ Third Division of Internal Medicine; ${ }^{5}$ Department of Pharmacy; ${ }^{6}$ Centre for Education on Hospital \\ Medicine, Faculty of Medicine, Shimane University, Japan
}

Received 3 October 2012; Final revision 15 January 2013; Accepted 1 February 2013; first published online 11 April 2013

\section{SUMMARY}

A seroprevalence survey on measles, mumps, rubella and varicella was conducted on healthcare workers (HCWs) at Shimane University Hospital, Japan utilizing an enzyme immunoassay. Of $1811 \mathrm{HCWs}$ tested, $91 \cdot 8 \%$ were seropositive to measles, $92 \cdot 1 \%$ to mumps, $89 \cdot 5 \%$ to rubella and $96.3 \%$ to varicella. Sex-related differences in seroprevalence were found in rubella (males vs. females: 84.7 vs. $92.2 \%, P<0 \cdot 001$ ). Moreover, males aged $30-39$ years were most susceptible to rubella $(22 \cdot 4 \%)$, which may be attributed to the design of childhood immunization programmes in Japan. Individuals aged $\leqslant 29$ years were more susceptible to measles $(14 \cdot 3 \%)$ and mumps $(10 \cdot 9 \%)$, compared to other age groups. There were no significant sex- and age-related differences in varicella seroprevalence. The physician occupational group was more susceptible to rubella, but no significant occupational-related difference was observed in the other diseases. Susceptible subjects, with negative or equivocal serological results were given a vaccine which induced seroconversion in most vaccinees. Seroconversion occurred more frequently in the equivocal group than in the negative group. These findings provide a new insight for the seroprevalence survey of vaccine-preventable diseases in Japanese HCWs with special reference to vaccine efficacy.

Key words: Healthcare workers, measles, mumps, rubella, seroprevalence survey, varicella, vaccination.

\section{INTRODUCTION}

Measles, mumps, rubella, and varicella, are transmissible viral infections that can be spread from an infected person to a susceptible person. These diseases are mediated via air droplets. Measles and varicella can also be mediated via airborne transmission. Measles

\footnotetext{
* Author for correspondence: S. Kumakura, M.D., Ph.D., Department of Medical Education and Research, Faculty of Medicine, Shimane University, Izumo, 693-8501, Japan. (Email: kumakura@med.shimane-u.ac.jp)
}

outbreaks occurred each year in Japan between 1999 and 2003, and recently Japan has experienced large measles outbreaks in 2007 and 2008 [1]. In the case of rubella, large outbreaks occurred in 1976, 1982, 1987 and 1992 in Japan, but the numbers of patients with rubella gradually decreased each year [2]. On the other hand, the occurrence of mumps and varicella in Japan is epidemic. The National Epidemiological Surveillance of Vaccine-Preventable Diseases in Japan has approximately 3000 paediatric and 450 adult sentinel institutes, which report numbers of patients with infectious diseases. The annual numbers of patients 
per institute in 2009 were $34 \cdot 6$ and $67 \cdot 09$ for mumps and varicella, respectively [2]. Thus, the control and prevention of these diseases in Japan remains critical.

Persons working in medical facilities have a greater risk of being exposed to and acquiring measles, mumps, rubella, and varicella. Outbreaks of these diseases have occurred not only in the community but also in the hospital setting [3]. Therefore, a seroprevalence survey for these diseases, followed by an adequate vaccination programme is necessary in the hospital setting. Vaccination programmes can substantially reduce both the number of susceptible healthcare workers (HCWs) and the attendant risks for transmission of vaccine-preventable diseases to other workers and patients [3, 4]. In Japan, vaccines for measles, mumps, rubella, and varicella, as well as hepatitis B and influenza, are offered to HCWs. Whether they are provided free of charge or not differs by hospital. For example, vaccines for hepatitis B, measles, mumps, rubella, and varicella are provided free of charge, while an influenza vaccine costs the individual about $£ 7$ at our university hospital.

Using enzyme immunoassay (EIA), we evaluated the immune status of HCWs at Shimane University Hospital, Japan, against measles, mumps, rubella, and varicella. In susceptible $\mathrm{HCWs}$ a vaccine was recommended, and the effects of the vaccine on acquisition of immunity were determined.

\section{SUBJECTS AND METHODS}

\section{Study population}

The subjects included in this study were HCWs at Shimane University Hospital, Japan. Since 2005, in order to control and prevent nosocomial measles, mumps, rubella and varicella, the infection control committee of Shimane University Hospital decided to survey the immune status of HCWs with vaccination being recommended for susceptible HCWs. In 2005 it was decided that all HCWs should receive serological screening for measles, mumps, rubella and varicella, and from 2006 screening was extended to include all newly employed HCWs. Our university hospital consists of the departments of endocrinology, gastroenterology, neurology, rheumatology, haematology, cardiology and respiratory medicine, gastrointestinal surgery, cardiovascular surgery, paediatrics, obstetrics and gynecology, orthopaedic surgery, neurosurgery, psychiatry, otorhinolaryngology - head and neck surgery, ophthalmology, urology, dermatology, radiology, anaesthesiology, emergency medicine and oral and maxillofacial surgery. HCWs enrolled in this study worked in the above-mentioned departments. Physicians, nurses, laboratory technicians, administrative staff and teaching staff were equally represented in the study. After informed consent, sera were collected between 2005 and 2009 from subjects. A structured questionnaire, including history of measles, mumps, rubella, and varicella infections and the status of vaccination records for these viruses was administered to HCWs enrolled in this study. This study was performed in the form of an audit as part of the hospital's safety and clinical service development.

\section{Assay for detecting virus antibodies}

For detecting the specific IgG antibodies against measles, mumps, rubella, and varicella by EIA, we used commercially available VIDAS assay kits; Measles-IgG, Mumps-IgG, RUB-IgG, and varicellazoster IgG (bioMérieux, France). The quantitative cut-off value for seronegativity was $<0.5$ for measles, $<0.35$ for mumps, $<10 \mathrm{IU} / \mathrm{ml}$ for rubella and $<0.6$ for varicella, and for titres equivocal values were defined as $0 \cdot 5-0 \cdot 7$ for measles, $0 \cdot 35-0 \cdot 50$ for mumps, $10-15 \mathrm{IU} / \mathrm{ml}$ for rubella and $0 \cdot 6-0 \cdot 9$ for varicella. The seropositive cut-off was $\geqslant 0 \cdot 7$ for measles, $\geqslant 0 \cdot 5$ for mumps, $\geqslant 15 \mathrm{IU} / \mathrm{ml}$ for rubella and $\geqslant 0.9$ for varicella. EIAs were conducted by the staff of the immunoserology unit of the Central Clinical Laboratory at Shimane University Hospital.

\section{Vaccination}

HCWs exhibiting a negative or equivocal antibody titre by EIA were considered as susceptible and recommended for vaccination. We used dried live-attenuated measles, mumps and rubella vaccine (Takeda Pharmaceutical Co. Ltd, Japan), and varicella vaccine (Tanabe Pharmaceutical Co. Ltd, Japan). Six months after the introduction of dried live-attenuated measles, rubella combined vaccine (Tanabe Pharmaceutical Co. Ltd) in 2007, its effect on vaccinated HCWs for the acquisition of immunity were investigated. HCWs, whose antibody titres remained negative or equivocal, were recommended for re-vaccination.

\section{Statistical analysis}

We used $\chi^{2}$ tests for the effects of sex, age, and occupation on seroprevalence. The interaction of these 
factors was tested in analysis of variance with $\chi^{2}$ distribution after angular transformation of seroprevalence. The statistically relevant criteria were set at $P<0 \cdot 05$.

\section{RESULTS}

\section{Characteristics of subjects}

Of the total number of HCWs, $89 \cdot 3 \%(912 / 1021)$ were surveyed in 2005 , and $89 \cdot 9 \%, 85 \cdot 8 \%, 79 \cdot 1 \%$ and $88 \cdot 5 \%$ of newly employed HCWs received serological screening in 2006, 2007, 2008 and 2009, respectively. Consequently, a total of 1811 HCWs $(86.9 \%$ of the total HCWs) were surveyed between 2005 and 2009, comprising 662 physicians, 622 nurses, 167 laboratory technicians, 295 administrative staff, and 65 teaching staff. There were 658 males and 1153 females. The age ranged from 19 to 64 years (mean \pm standard deviation $34 \cdot 3 \pm 10 \cdot 2$ ). The mean length of employment of enrolled HCWs, who worked at the university hospital in 2005 , was 10.5 years (mean \pm standard deviation, $10 \cdot 5 \pm 10 \cdot 0$ ). The characteristics of HCWs are given in Table 1. The questionnaire, including history of diseases and vaccination status, demonstrates invalid data as the majority of HCWs did not remember their disease history nor did they have reliable vaccination records.

\section{Seroprevalence of virus antibodies in HCWs}

Of the HCWs tested, $91 \cdot 8 \%$ were seropositive to measles, $92 \cdot 1 \%$ to mumps, $89 \cdot 5 \%$ to rubella and $96.3 \%$ to varicella (Table 2 ). The annual prevalence rate of seropositivity to these viruses between 2005 and 2009 is given in Table 3, demonstrating similar seroprevalence during this period.

No significant difference was found between male and female HCWs with regard to seropositivity to measles, mumps, and varicella; however, male HCWs were less seropositive to rubella than female HCWs (84.7 vs. $92 \cdot 2 \%, P<0 \cdot 001)$.

Next, we analysed data according to age. There were significant differences in seropositivity rates in age groups for measles, mumps, and rubella. For measles, younger age groups were less seropositive than older age groups. Those aged $\leqslant 29$ years were less seropositive than those aged $30-39$ years $(85 \cdot 7 \%$ vs. $93.2 \%, P<0.001)$. The $30-39$ years age group was also less seropositive than the 40-49 years age group $(93.2 \%$ vs. $99 \cdot 2 \%, P<0 \cdot 001)$. Similarly, for mumps, younger age groups were less seropositive
Table 1. Characteristics of the study subjects

\begin{tabular}{lcl}
\hline \hline Characteristic & No. & $(\%)$ \\
\hline Healthcare workers tested & 1811 & \\
Sex & & \\
$\quad$ Male & 658 & $(36)$ \\
$\quad$ Female & 1153 & $(64)$ \\
Age (yr) & & \\
$\quad \leqslant 29$ & 763 & $(42)$ \\
$30-39$ & 498 & $(28)$ \\
$40-49$ & 379 & $(21)$ \\
$\geqslant 50$ & 171 & $(9)$ \\
Length of employment (yr)* & & \\
$<5$ & 440 & $(50)$ \\
$5-9$ & 86 & $(10)$ \\
$10-19$ & 132 & $(15)$ \\
$20-29$ & 212 & $(24)$ \\
$\geqslant 30$ & 11 & $(1)$ \\
Occupation & & \\
Physician & 662 & $(37)$ \\
Nurse & 622 & $(34)$ \\
Technician & 167 & $(9)$ \\
Administrator & 295 & $(16)$ \\
Teaching staff & 65 & $(4)$ \\
\hline \hline
\end{tabular}

* Data from healthcare workers employed at the university hospital in 2005.

Table 2. Serological results of 1811 healthcare workers tested

\begin{tabular}{lllllrl}
\hline \hline & \multicolumn{2}{l}{$\begin{array}{l}\text { Positive, } \\
n(\%)\end{array}$} & \multicolumn{2}{l}{$\begin{array}{l}\text { Equivocal, } \\
n(\%)\end{array}$} & \multicolumn{2}{l}{$\begin{array}{l}\text { Negative, } \\
n(\%)\end{array}$} \\
\hline Measles & 1663 & $(91 \cdot 8)$ & 62 & $(3 \cdot 5)$ & 86 & $(4 \cdot 7)$ \\
Mumps & 1667 & $(92 \cdot 1)$ & 33 & $(1 \cdot 8)$ & 97 & $(6 \cdot 1)$ \\
Rubella & 1620 & $(89 \cdot 5)$ & 27 & $(1 \cdot 5)$ & 164 & $(9)$ \\
Varicella & 1744 & $(96 \cdot 3)$ & 45 & $(2 \cdot 5)$ & 22 & $(1 \cdot 2)$ \\
\hline \hline
\end{tabular}

than older age groups. Those aged $\leqslant 29$ years were less seropositive than the 30-39 years $(89 \cdot 1 \%$ vs. $92 \cdot 6 \%, P<0.05)$ and $40-49$ years $(89 \cdot 1 \%$ vs. $95 \cdot 0 \%$, $P<0.01)$ age groups. While younger age groups showed a higher seropositivity rate to rubella than older age groups, the seropositivity rate was significantly different between the $\leqslant 29$ years and the $30-39$ years age groups $(94 \cdot 2 \%$ vs. $85 \cdot 5 \%, P<0 \cdot 001)$. Rubella seropositivity rates in the 30-39, 40-49 and $>50$ years age groups were most similar $(85.5 \%$, $86 \cdot 8 \%$ and $85 \cdot 6 \%$, respectively). There were no significant age-related differences in the prevalence of immunity for varicella, and $~ 95 \%$ of $\mathrm{HCWs}$ were seropositive [ $\leqslant 29$ years $(94 \cdot 9 \%), 30-39$ years $(97 \cdot 6 \%)$, $40-49$ years $(97 \cdot 4 \%), \geqslant 50$ years $(96 \cdot 0 \%)]$. 
Table 3. Seropositivity rate (\%) of healthcare workers between 2005 and 2009

\begin{tabular}{lccccc}
\hline \hline & 2005 & 2006 & 2007 & 2008 & 2009 \\
\hline Measles & $94 \cdot 0$ & $88 \cdot 7$ & $85 \cdot 1$ & $85 \cdot 1$ & $92 \cdot 3$ \\
Mumps & $93 \cdot 2$ & $87 \cdot 0$ & $87 \cdot 0$ & $86 \cdot 7$ & $90 \cdot 1$ \\
Rubella & $86 \cdot 5$ & $89 \cdot 5$ & $87 \cdot 4$ & $83 \cdot 2$ & $89 \cdot 5$ \\
Varicella & $97 \cdot 6$ & $95 \cdot 4$ & $93 \cdot 0$ & $95 \cdot 6$ & $88 \cdot 4$ \\
\hline \hline
\end{tabular}

Based on these findings, we further analysed sexrelated differences for different age groups. Although there were no significant sex-related differences in any age group for measles, mumps, and varicella, rubella seropositivity rates differed markedly between the sexes at a younger age ( $<39$ years). For individuals aged $\leqslant 29$ years, male subjects exhibited lower seropositivity rates than females $(89.5 \mathrm{vs} .95 .7 \%, P<0.01)$. In the 30-39 years age group, the seropositivity rate was markedly low in males compared to females (77.6 vs. 93.4, $P<0.001)$. Male seropositivity in the 30-39 years age group was significantly lower than for those aged $\leqslant 29$ years $(77 \cdot 6 v s .89 \cdot 5 \%, P<0 \cdot 01)$, as well as for those aged $40-49$ years $(77.6 \mathrm{vs}$. $88 \cdot 1 \%, P<0 \cdot 01)$.

In addition, we examined occupational-related differences, which revealed no significant differences for occupations in measles, mumps, and varicella. However, for rubella, the physician group was less seropositive than the other groups: nurses (86.4 vs. $91 \cdot 2 \%$, $P<0 \cdot 01$ ), technicians ( 86.4 vs. 92.8\%, $P<0.05$ ), administrators $(86 \cdot 4$ vs. $91 \cdot 2 \%, P<0 \cdot 05)$. Sex-related differences in occupation were also observed. In the physician group, males were more prevalent than females (males vs. females: $68 \%$ vs. $32 \%$ ), while in the other occupational groups females were more prevalent or equal to males [males vs. females: nurses ( $6 \%$ vs. $94 \%$ ), technicians ( $50 \%$ vs. $50 \%$ ), administrators (29\% vs. $71 \%)$ ].

We also investigated differences between departments in immunity to the different antigens by analysing physicians' seroprevalence, which revealed no significant difference between departments in seroprevalence to the different antigens (data not shown).

\section{Vaccination and acquisition of immunity}

Between 2005 and 2009, a total of 61, 59, 70 and 18 susceptible HCWs received vaccination for measles, mumps, rubella, and varicella, respectively. Of 61 subjects vaccinated for measles, 22 had equivocal values and 39 had negative values before vaccination. In the equivocal group $(n=22), 21$ subjects seroconverted, but one subject remained with an equivocal value. The latter subject was re-vaccinated, which successfully induced seroconversion. In the negative group $(n=39)$, seroconversion was achieved by 33 subjects. The remaining six subjects had equivocal $(n=2)$ and negative $(n=4)$ values. Of these remaining six subjects, one who had an equivocal value and three who had negative values were re-vaccinated, resulting in seroconversion for three subjects. The remaining subject, who remained negative after re-vaccination, received a third vaccination with a successful outcome.

Regarding mumps, initial vaccination induced seroconversion in 11 subjects in the equivocal group $(n=12)$ and in 27 subjects in the negative group $(n=47)$. The remaining subjects in the negative group exhibited equivocal $(n=7)$ and negative $(n=13)$ values. Of the subjects who remained with an equivocal value $(n=7)$, three were re-vaccinated, and all seroconverted. Of the subjects who remained with a negative value after the initial vaccination $(n=13)$, nine were re-vaccinated. Consequently, five of these seroconverted, three had an equivocal value and one had a negative value. Finally, one subject who remained with an equivocal value, and one subject with a negative value received a third vaccination. Both achieved seroconversion.

Regarding rubella, 70 susceptible subjects were vaccinated. In the equivocal group $(n=4)$, three subjects achieved seroconversion, and one remained with an equivocal value. The latter subject subsequently seroconverted following a third vaccination. In the negative group $(n=66), 46$ subjects seroconverted, while 10 remained with an equivocal value and 10 remained with a negative value. Seven of these 10 subjects with an equivocal value after the initial vaccination were re-vaccinated, which resulted in five seroconverting; however, two remained with an equivocal value. Four out of 10 subjects with a negative value after the initial vaccination were re-vaccinated. Of these, one subject exhibited an equivocal value while the other three exhibited negative values. Subsequently, two subjects received a third vaccination, without seroconversion.

Eighteen susceptible subjects for varicella received vaccination. All nine subjects in the equivocal group achieved seroconversion. In the negative group $(n=9)$, four subjects seroconverted, one remained with an equivocal value and four subjects had a negative 
Table 4. Effects of vaccination on seroconversion rate in susceptible healthcare workers

\begin{tabular}{|c|c|c|c|c|c|c|c|c|}
\hline & \multicolumn{8}{|c|}{ Seroconverted/vaccinated $(N / n, \%)$} \\
\hline & \multicolumn{2}{|c|}{ Measles } & \multicolumn{2}{|c|}{ Mumps } & \multicolumn{2}{|c|}{ Rubella } & \multicolumn{2}{|c|}{ Varicella } \\
\hline \multicolumn{9}{|c|}{ Initial vaccination } \\
\hline Negative $†$ & $33 / 39$ & $(84 \cdot 6 \%)$ & $27 / 47$ & $(57 \cdot 4 \%)$ & $46 / 66$ & $(69 \cdot 7 \%)$ & $4 / 9$ & $(44 \cdot 4 \%)$ \\
\hline Equivocal $\dagger$ & $21 / 22$ & $(95 \cdot 5 \%)$ & $11 / 12$ & $(91 \cdot 7 \%)^{*}$ & $3 / 4$ & $(75 \%)$ & 9/9 & $(100 \%)^{* *}$ \\
\hline Total & $54 / 61$ & $(88 \cdot 5 \%)$ & $38 / 59$ & $(64 \cdot 4 \%)$ & $49 / 70$ & $(70 \%)$ & $13 / 18$ & $(72 \cdot 2 \%)$ \\
\hline \multicolumn{9}{|l|}{ Re-vaccination } \\
\hline Negative† $†$ & $2 / 3$ & $(66 \cdot 7 \%)$ & $5 / 9$ & $(55 \cdot 6 \%)$ & $0 / 3$ & $(0 \%)$ & $0 / 1$ & $(0 \%)$ \\
\hline Equivocal $\dagger$ & $2 / 2$ & $(100 \%)$ & $3 / 3$ & $(100 \%)$ & $6 / 8$ & $(75 \%)$ & $1 / 1$ & $(100 \%)$ \\
\hline Total & $4 / 5$ & $(80 \%)$ & $8 / 12$ & $(66 \cdot 7 \%)$ & $6 / 11$ & $(54 \cdot 5 \%)$ & $1 / 2$ & $(50 \%)$ \\
\hline
\end{tabular}

$\dagger$ Enzyme immunoassay value of healthcare workers before vaccination.

* $P<0.05,{ }^{*} P<0.01$ relative to negative value group.

value. One subject with an equivocal value was re-vaccinated, which induced seroconversion. One subject with a negative value failed to respond to revaccination.

We estimated the overall seroconversion rate, which is shown in Table 4. The overall seroconversion rate induced by initial vaccination was $88.5 \%$ for measles, $64 \cdot 4 \%$ for mumps, $70 \%$ for rubella and $72 \cdot 2 \%$ for varicella. The re-vaccination induced seroconversion rate was $80 \%$ for measles, $66 \cdot 7 \%$ for mumps, $54 \cdot 5 \%$ for rubella and $50 \%$ for varicella. The effects of vaccination on seroconversion differed between the equivocal and the negative groups. The equivocal group revealed a higher seroconversion rate by initial vaccination than the negative group $[95 \cdot 5 \%$ vs. $84 \cdot 6 \%$ for measles (not significant), $91 \cdot 7 \%$ vs. $57 \cdot 4 \%$ for mumps $(P<0 \cdot 05), 75 \%$ vs. $69 \cdot 7 \%$ for rubella (not significant), $100 \%$ vs. $44 \cdot 4 \%$ for varicella $(P<0 \cdot 001)]$. After re-vaccination, high seroconversion rates in the equivocal group compared to the negative group were also noted.

\section{DISCUSSION}

In this study we tested sera, using EIA, collected from a total of $1811 \mathrm{HCWs}$ at a tertiary-care hospital in Japan, and found that $91.8 \%$ were seropositive to measles, $92 \cdot 1 \%$ to mumps, $89 \cdot 5 \%$ to rubella and $96 \cdot 3 \%$ to varicella. These results are similar to results obtained with studies performed in HCWs in Japan $[5,6]$. On the other hand, similar studies from other regions revealed that in Australia $91.5 \%$ of $\mathrm{HCWs}$ were seropositive to measles, $88.7 \%$ to mumps, $91 \cdot 1 \%$ to rubella and $89 \cdot 1 \%$ to varicella [7]. In Italy and Turkey, $98 \cdot 2 \%$ and $98 \cdot 6 \%$ were seropositive to measles, $85.9 \%$ and $92.2 \%$ to mumps, $97.6 \%$ and $98.3 \%$ to rubella and $97 \cdot 9 \%$ and $98 \%$ to varicella, respectively [8, 9]. In Saudi Arabia, seropositivity rates were shown to be $87 \%$ to measles, $90 \%$ to rubella and $86 \%$ to varicella [10]. There are some differences between regional seropositivity rates which are perhaps attributable to differences in the design of early childhood immunization programmes of each country.

In Japan, vaccination programmes for measles and mumps began in 1966 and 1981, respectively. In 1989, a measles-mumps-rubella (MMR) vaccine was introduced, and was subsequently terminated in 1993. Since 2006, a routine measles-rubella (MR) vaccine was introduced; however, the mumps vaccine continues to be excluded from the legal vaccine. Against this background, subjects susceptible for measles and mumps could be found specifically in the younger population (aged $\leqslant 29$ years) in Japan $[11,12]$, which corresponds to our findings that the population of HCWs aged $\leqslant 29$ years was most susceptible for measles and mumps. Immunization for these susceptible HCWs is important not only to prevent nosocomial transmission of these diseases but also to ultimately eliminate them. Japan set a target date of 2012 for measles elimination and has promoted measles control strategies since 2001, when the largest measles outbreak occurred in Japan [1].

Our survey revealed that males were more susceptible for rubella than females, and males aged 30-39 years were most susceptible for rubella $(22.4 \%)$. The age- and sex-related differences in the prevalence of susceptibility to rubella in HCWs may be explained 
by the late initiation of vaccination and previous history of disease in male subjects. The widespread occurrence of rubella has been noted in Japan in 1966, after which rubella occurred as an epidemic. However, since 1980, despite some random outbreaks, the annual numbers of patients with rubella gradually decreased every year, owing to the effects of vaccination programmes. Routine childhood vaccination for rubella began in 1977, given only to female junior high school students (12-15 years). MMR vaccination was introduced in 1989, and was terminated in 1993. Since 1994, the monovalent rubella vaccine was recommended for both male and female infants (1-7.5 years), and MR vaccine was initiated in 2006. Thus, HCWs aged $\geqslant 40$ years, who were born before 1976, had the possibility of suffering during the rubella epidemic, while those aged $<40$ years were less likely to do so. These findings would explain agedependent difference in the prevalence of rubella antibodies observed in our survey. In the 30-39 years age group, males demonstrated a higher prevalence of susceptibility to rubella than females. This may be attributable to vaccine programmes, in which only females received rubella vaccines during 19971988. Since 1989, males, as well as females, also received the vaccine, resulting in a lower prevalence of susceptibility in males aged $\leqslant 29$ years than in those aged 30-39 years.

The varicella vaccine developed in Japan in the 1970s was licensed in Japan since 1987. However, the varicella vaccine was not compulsory, and therefore coverage among infants has increased only from $6 \cdot 8 \%$ at introduction in 1987 to $32 \cdot 1 \%$ in 2005 [13]. Over $90 \%$ of unvaccinated persons become infected, and over $80 \%$ of persons have been infected by age 10 years in Japan, which has resulted in high seroconversion rates. This evidence is consistent with our finding that there is a high varicella seropositivity rate in HCWs with no significant sex- or age-related differences.

We also found occupational-related difference in seroprevalence of rubella antibodies. In the physician group, the majority $(76 \cdot 8 \%)$ of subjects were relatively young (aged 20-39 years), with males being more prevalent, which may explain the reason why the physician group demonstrated a notably higher prevalence of susceptibility to rubella, since both relative youth (aged 20-39 years) and male sex attribute to high susceptibility rates to rubella in Japan [14]. We believe that age and sex factors can be attributable to occupational-related differences; however, occupation is a confounding factor and further examination is needed.

Total seroconversion rates by initial vaccination were estimated as $88.5 \%$ for measles, $64.4 \%$ for mumps, $70 \cdot 0 \%$ for rubella and $72 \cdot 2 \%$ for varicella. Subjects whose antibody titres remained negative or equivocal were subsequently re-vaccinated, which resulted in seroconversion in $95 \cdot 3 \%, 85 \cdot 5 \%, 87 \cdot 2 \%$ and $70 \cdot 6 \%$ of subjects for measles, mumps, rubella, and varicella, respectively. Asari et al. have reported that seroconversion rates in susceptible $\mathrm{HCW}$ were low for rubella $(50 \%)$, intermediate for measles and mumps $(80 \%)$ and high for varicella $(100 \%)$, while re-vaccination for those remaining seronegative resulted in 100\% seroconversion [6]. In another study of medical students, high seroconversion rates were noted: $100 \%$ for measles and rubella, $89 \%$ for mumps, and $67 \%$ for varicella [15]. The differences in seroconversion rates in these studies may depend, at least in part, on the differences in the vaccine preparations on the market.

It remains unclear whether individuals who were re-vaccinated and failed to seroconvert did not do so due to other reasons. These re-vaccinated individuals who failed to seroconvert did not have any evidence of underlying immunocompromised conditions. There were no subjects who were undergoing immunosuppressive therapy, had malignant diseases, or were immunodeficient. Among measles, mumps, rubella, and varicella antibodies, individuals who were revaccinated and did not seroconvert were positive to at least one antibody, which suggests that antibody response is not completely impaired in these individuals. A recent study identified variants of CD46 and Toll-like receptor 8 , which are critically involved in the recognition of vaccine strains of the measles virus, in measles vaccine failure [16]. Although these genetic variants might be associated with the occurrence of vaccine failure in some cases, in our adult cases, mechanisms mediating the inhibition of vaccineinduced antibody response remain unclear and should be further investigated.

Nevertheless, our results show that the majority of vaccinees seroconverted by the third vaccination, indicating the effectiveness of vaccination in susceptible HCWs. The prevention of these diseases through comprehensive personnel immunization programmes is far more cost-effective than case management and outbreak control [4].

Interestingly, we observed a difference in seroconversion rates between individuals with an equivocal 
value and a negative value. Seroconversion was more frequently seen in the equivocal group than in the negative group: $95 \cdot 5 \%$ vs. $84 \cdot 6 \%$ for measles, $91 \cdot 7 \%$ vs. $57 \cdot 4 \%$ for mumps, and $75 \%$ vs. $69 \cdot 7 \%$ for rubella, and $100 \%$ vs. $44 \cdot 4 \%$ for varicella. One study surveying the seroprevalence of measles in seven countries in Western Europe indicated that the proportion of the equivocal group was high in vaccinated age groups [17]. Therefore, high seroconversion rates in equivocal groups may be due to the booster effects of a considerable number of subjects, whose titres have waned to low positive levels (equivocal values) after previous vaccination. Antigen-specific antibody responses can be boosted through vaccination, since memory B cells in circulation are markedly long-lived [18]. Primary immune response to the first immunization during childhood provides long-term protection.

Antibody threshold levels that provide protection from these diseases seem not to be well standardized in the EIA system. Lee et al. examined the protective threshold of measles neutralizing antibody using neutralization enzyme immunoassay (NT-EIA) which employed EIA to detect the growth of a wild-type measles virus in Vero cells, and suggests that neutralizing titres $>1000 \mathrm{mIU} / \mathrm{ml}$ may prevent measles infection [19]. However, it is not clear how this antibody titre corresponds to the levels measured by each commercially available EIA. Tharmaphornpilas et al. calibrated the test using the National Substandard of Anti-Measles-Serum, Human, provided by the Robert Koch Institute, and a set cut-off point at $255 \mathrm{mIU} / \mathrm{ml}$ for measles IgG [20]. Amanna et al. used a measles protective titre of $200 \mathrm{mIU} / \mathrm{ml}$ [18]. However, in our EIA system (VIDAS assay), it remains unclear what the correspondence of cut-off values are for measles in $\mathrm{IU} / \mathrm{ml}$, and this therefore needs further clarification. On the other hand, in the case of varicella, a recent report has shown that a seropositive cut-off value of 0.9 in the VIDAS assay corresponds to $275-280 \mathrm{mIU} / \mathrm{ml}$ [21]. For rubella $\mathrm{IgG}$, the cut-off point in the USA is $10 \mathrm{IU} / \mathrm{ml}$ [22], while the common European threshold is $15 \mathrm{IU} / \mathrm{ml}$ [23]. In our survey, we defined the rubella equivocal value as $10-15 \mathrm{IU} / \mathrm{ml}$, based on the manufacturer's thresholds. Epidemiological evidence indicated that the $10 \mathrm{IU} / \mathrm{ml}$ antibody level is protective in most people [22], which suggests that the equivocal value in our survey appears to provide for a high threshold. In addition, EIA is known to have a lower sensitivity than the neutralization test and tends to overestimate equivocal and negative results [24, 25]. Thus, there is a need to ensure the validity of the rubella equivocal value of $10-15$ IU/ml. The standardization of antibody threshold levels is crucial to the determination of adequately susceptible subjects. To date, the antibody threshold for protection against mumps and varicella has not been determined. Other predictive markers indicating memory and effector functions should also be investigated because the immune status of individuals cannot be determined solely by humoral immunity.

In conclusion, we have shown the seroprevalence of a total of $1811 \mathrm{HCWs}$ against measles, mumps, rubella, and varicella, with special reference to vaccine efficacy. The seroprevalence survey had important implications for the management of vaccine programmes of HCWs, which contributes to the prevention of disease transmission in the hospital setting.

Unsolved problems remain, e.g. the standardization of antibody thresholds in different EIA systems, and the establishment of an efficient vaccination strategy for HCWs. However, despite the remaining problems, understanding the immune status of HCWs, followed by the promotion of vaccination, may provide for a higher qualification and safety in patient care. Last, further development of ongoing research is required, for the control, prevention, and elimination of vaccinepreventable diseases.

\section{ACKNOWLEDGEMENTS}

The authors thank Dr K.Taketani for helpful comments on the manuscript.

\section{DECLARATION OF INTEREST}

None.

\section{REFERENCES}

1. Centers for Disease Control and Prevention. Progress toward measles elimination - Japan, 1999-2008. Morbidity and Mortality Weekly Report 2008; 57: 1049-1052.

2. Infectious Disease Surveillance Center. (http://www. nih.go.jp/niid/ja/idwr/2085-ydata/1617-report-jb.html) [in Japanese]. Accessed 21 December 2012.

3. Centers for Disease Control and Prevention. Immunization of healthcare workers: recommendation of the Advisory Committee on Immunization Practices (ACIP) and the Hospital Infection Control Practices Advisory Committee (HICPAC). Morbidity and Mortality Weekly Report. Recommendations and Reports 1997; 46: 1-42.

4. Bolyard EA, et al. Guideline for infection control in healthcare personnel, 1998. Hospital Infection Control Practices Advisory Committee. Infection Control and Hospital Epidemiology 1998; 19: 407-463. 
5. Hatakeyama S, et al. Prevalence of measles, rubella, mumps, and varicella antibodies among healthcare workers in Japan. Infection Control and Hospital Epidemiology 2004; 25: 591-594.

6. Asari S, et al. Seroprevalence survey of measles, rubella, varicella, and mumps antibodies in health care workers and evaluation of a vaccination program in a tertiary care hospital in Japan. American Journal of Infection Control 2003; 31: 157-162.

7. Vagholkar S, et al. Healthcare workers and immunity to infectious diseases. Australian and New Zealand Journal of Public Health 2008; 32: 367-371.

8. Fedeli U, Zanetti C, Saia B. Susceptibility of healthcare workers to measles, mumps rubella and varicella. Journal of Hospital Infection 2002; 51: 133-135.

9. Celikbas A, et al. Measles, rubella, mumps, and varicella seroprevalence among health care workers in Turkey: is prevaccination screening cost-effective? American Journal of Infection Control 2006; 34: 583587.

10. Almuneef MA, et al. Seroprevalence survey of varicella, measles, rubella, and hepatitis A and B viruses in a multinational healthcare workforce in Saudi Arabia. Infection Control and Hospital Epidemiology 2006; 27: 1178-1183.

11. Infectious Disease Surveillance Center. Measles, Japan, 2006-2007. (http://idsc.nih.go.jp/iasr/28/331/tpc331.html). Accessed 15 September 2012.

12. Infectious Disease Surveillance Center. Measles in Japan, 2009. (http://idsc.nih.go.jp/iasr/31/360/tpc360. html). Accessed 15 September 2012.

13. Sadzot-Delvaux C, et al. Varicella vaccination in Japan, South Korea, and Europe. Journal of Infectious Diseases 2008; 197 (Suppl. 2): 185-190.

14. Infectious Disease Surveillance Center. (http://idsc.nih. go.jp/yosoku/Rubella/Serum-R2009-e.html). Accessed 21 December 2012.

15. Kaetsu A, et al. An outbreak of measles in Saitama City in 2007. What is the vaccination strategy to eliminate measles in Japan? Journal of Infection and Chemotherapy 2008; 14: 291-295.
16. Clifford HD, et al. Polymorphisms in key innate immune genes and their effects on measles vaccine responses and vaccine failure in children from Mozambique. Vaccine 2012; 30: 6180-6185.

17. de Melker H, et al. The seroepidemiology of measles in Western Europe. Epidemiology and Infection 2001; 126: 249-259.

18. Amanna IJ, Carlson NE, Slifka MK. Duration of humoral immunity to common viral and vaccine antigens. New England Journal of Medicine 2007; 357: 1903-1915.

19. Lee MS, et al. Protective titres of measles neutralising antibody. Journal of Medical Virology 2000; 62: 511517.

20. Tharmaphornpilas $\mathbf{P}$, et al. Seroprevalence of antibodies to measles, mumps, and rubella among Thai population: evaluation of measles/MMR immunization programme. Journal of Health, Population and Nutrition 2009; 27: 80-86.

21. Maple PA, et al. Comparison of the performance of the LIAISON VZV-IgG and VIDAS automated enzyme linked fluorescent immunoassays with reference to a VZV-IgG time-resolved fluorescence immunoassay and implications of choice of cut-off for LIAISON assay. Journal of Clinical Virology 2009; 44: 9-14.

22. Skendzel LP. Rubella immunity. Defining the level of protective antibody. American Journal of Clinical Pathology 1996; 106: 170-174.

23. Matter L, Kogelschatz K, Germann D. Serum levels of rubella virus antibodies indicating immunity: response to vaccination of subjects with low or undetectable antibody concentrations. Journal of Infectious Diseases 1997; 175: 749-755.

24. Backhouse JL, et al. Evaluation of two enzyme immunoassays for detection of immunoglobulin $\mathrm{G}$ antibodies to mumps virus. Clinical and Vaccine Immunology 2006; 13: $764-767$.

25. Davidkin I, et al. Persistence of measles, mumps, and rubella antibodies in an MMR-vaccinated cohort: a 20-year follow-up. Journal of Infectious Diseases 2008; 197: 950-956. 